\title{
Tool selection for public diplomacy flagships: toward an adaptive model
}

\author{
Biyun Zhu ${ }^{1}$ (D)
}

Revised: 6 May 2021 / Accepted: 21 May 2021 / Published online: 4 June 2021

(c) The Author(s), under exclusive licence to Springer Nature Limited 2021

\begin{abstract}
The evolving relationship between nation-states and sub-national actors makes public policy a meaningful inside-out approach in public diplomacy (PD) research. This multi-level participation and complexity have also drawn attention to "flagship" as a particular mechanism that systematically coordinates and manages PD activities. Public diplomacy flagship, such as the Fulbright Program and the British Council, is an orchestra of various programs that serves the national agenda as well as personal and organizational goals. Yet, we seem to know little about the decision-making process of why the programs are selected and how they are fitted into a flagship. This article attempts to address this question from a public policy perspective. Viewing PD activities in various kinds as "tool options," this article presents an adaptive model of tool selection to unpack the decision-making in public diplomacy flagships. Acknowledging the influence of human factors and the risk of over-generalization, the model aims to present one possibility among many and focus on the underlying relationships between the tools and their roles in a flagship. With examples from the Fulbright Program, the British Council, and Confucius Institute, this article explains how different users can adapt the model for their analytical, evaluative, and comparative purposes.
\end{abstract}

Keywords Public diplomacy $\cdot$ Flagships $\cdot$ Public policy $\cdot$ Tool selection

\section{Introduction}

As an interdisciplinary field, public diplomacy (PD) has attracted scholars of several disciplines (e.g., communication and international relations) who have contributed theories that further our understanding and better the practice. In comparison, the discipline of public policy seems to be a less popular perspective for the perceived distinction between domestic and foreign policies. However, with the increasing participation of non-state and sub-national actors at multiple levels such as local and civic organizations (Melissen and Wang 2019), and the entanglement of domestic and global environments (Hocking et al. 2013), this distinction non longer sustains. The activities, resources, and governing methods of PD are shaped by a wide range of domestic policies in the areas of education, culture, broadcasting, etc. The blurring boundaries and increasing multi-level participation make public policy an interesting

Biyun Zhu

zhu.1665@osu.edu

1 The Ohio State University, 231 Sullivant Hall, 1813 N High Street, Columbus, OH 43210, USA inside-out approach to examine PD practice in addition to the disciplines of international relations and communication. Public policy could contribute to PD scholarship by focusing on the decision-making process and articulating the characteristics and dynamics of PD activities.

While PD has become a statecraft of many nations, it appears as a more organized and systematic approach in some countries than in others. The flagship of public diplomacy is such a systematic endeavor to integrate PD activities through strategic planning, governance, and resource optimization to enhance international influence and advance national interests. Examples of public diplomacy flagships include the British Council, the Fulbright Program, and Confucius Institute. Each of these flagships, technically, is not a single government program but is a structure, a mechanism, and an umbrella that holds up and coordinates a wide range of activities. Each of these programs is perceived as a tool/ tool mix in public policy which has its own attributes and relative advantages and disadvantages, which allows policy makers to combine and balance their choices. By developing a portfolio of tools that is legitimate, flexible, and sustainable, flagships have unique strengths in an increasingly competitive and volatile international environment. However, we 
know little about the attributes and relationships of these tools in flagship: how decisions are made, and what contributes to better practice. Targeting the tool selection question and acknowledging the influences of human factors and the risk of over-generalization, this article develops an adaptive model that includes a strategic toolkit and a strategic menu to interpret how PD tools are put together as a flagship to advance national purposes in different contexts.

\section{Public diplomacy flagships: definition and distinctions}

According to Merriam-Webster Dictionary, flagship is defined as the finest, largest, or most important one of a group of things. In cultural and urban policy, the word has been frequently used on local cultural organizations and projects that play a vital role in urban development and place branding (Bianchini et al. 1992; Smyth 2005; De Frantz 2005; Grodach 2010). These local cultural flagship projects include arts complexes and iconic buildings that hold reputable museums and performing arts companies, which become an important name card of the city. The Guggenheim Museum at Bilbao is one of such renowned cases. These cultural flagships serve as a catalyst to promote city image, boost tourism and commercial activities, and generate wealth, which shares a similar mechanism to public diplomacy. This similar mechanism and the link between place branding and public diplomacy is also elaborated by Anholt (2006), which marks the repositioning of this journal from Place Branding to Place Branding and Public Diplomacy.

At the national and transnational levels, flagship is also an appropriate word to describe PD programs for several reasons. First, similar to local flagship cultural projects, public diplomacy flagship is also a structure to hold up a variety of activities. For example, the Fulbright Program is a flagship because it is not a single educational exchange program but multiple programs that operated by several organizations ${ }^{1}$ serving diverse populations with different needs in the US and beyond. The British Council is a flagship because, in addition to language programs, it is active in other fields such as heritage preservation, partnership facilitation, and capacity development. The multiplicity and diversity of the activities enables the flagship to maneuver in a fluid and chaotic environment with flexibility and resilience. This observation does not suggest multiplicity is superior to simplicity, but rather highlights that it is a coping mechanism

\footnotetext{
${ }^{1}$ For example, stakeholders include but are not limited to U.S. State Department, U.S. Department of Education, and The Institute of International Education (IIE).
}

developed to address the unique challenges faced by public diplomacy flagships and nation-states in a changing environment. Second, similar to local cultural flagships as stimuli to urban regeneration, public diplomacy flagships function as a catalyst of national branding and open up a space for further communication and collaboration among countries. As the "signature" or the "face" of a country, a public diplomacy flagship has important symbolic importance for the country's culture, values, and foreign policy. It is the forefront where culture, information, and ideas interchanges and people familiarize themselves with another country. As a national brand, public diplomacy flagship should be inclusive but also somehow selective and representative of these symbols.

Finally, the strategic importance of the flagship distinguishes it from general PD activities in two dimensions. (1) The unique institutional setting of the flagship mobilizes great government support. The annual government spending on the flagships were over $\$ 200$ million in the U.S., UK, and China. ${ }^{2}$ For flagships, government presence is as important as public participation, and their co-existence legitimizes and authorizes the governance of the flagship. (2) The flexible structure and symbolic meaning of the flagship allow it to communicate efficiently and respond deftly to the changing environment with soft measures. History reveals that flagships serve the strategic role to position nation-states in a changing world. The British Council was established when the UK faced a decline in international influence as a result of the WWI, Great Recession, and decolonization in the 1930s. The Fulbright Program was created in 1946 when the U.S. faced the challenge to legitimize its new leadership in the post-war and Cold War era. Chinese Confucius Institute was created in 2004 against the backdrop of China threat theory, and the flagship was expected to justify China's greater role in the world at the beginning of the twenty-first century. Since the beginning, the governance of these flagships has been constantly challenged and adapted to international changes until today.

The above discussion leads to a definition of public diplomacy flagship: it represents a mechanism coordinated by government to select and manage a wide range of PD tools to address the multiple purposes of various actors, adapt to micro conditions as well as serve diverse audiences, which ultimately shapes the international environment to advance national interests.

\footnotetext{
2 The British Council reported $£ 184$ million (about \$254 million) government grant-in-aid in the 2018-2019 annual report (British Council 2019a). The Fulbright Program reported a \$236 million congressional appropriation in fiscal year 2016 (FFSB 2017). Confucius Institute reported $\$ 314$ million government funding in the 2016 annual report (Hanban 2016).
} 


\section{Understanding the tool selection of public diplomacy: what makes it difficult}

From public policy perspective, activities of different kinds (including traditional diplomatic and para-diplomacy activities) as options for a flagship can be considered as "policy tools". These policy tools are defined, packed, and organized under the framework of flagship serving national goals. The central questions then become: Why are certain tools selected, and how do they fit together to form a flagship? We seem to know little about how policy tools are selected in public diplomacy (Gilboa 2008). Existing literature has focused on the content, outcome and mechanism of a specific PD tool, such as international exchange (Pacher 2018) and new media (Sevin and Ingenhoff 2018), but few research have examined the process to select the tools and why such process is followed. This is first because the research is often challenged by subjective factors in decision-making, which is difficult to capture but critical to understand the tool selection process. Many decisions are made based on experience and attention to nuances that are not captured by rigid parameters in theories. As Pamment (2014) argues, PD decisions are often not rationalized due to the complex organizational and power structure. Second, what complicate the tool selection questions in PD are the variances across countries, organizations, and individuals. The dissimilar contexts, interests, and capacities make it difficult to explain tool selection of PD with a universal model. These variances could make the same tool effective in one country but failing in another. Because of these challenges, we constantly run the risks of over-generalization and simplification. While the exploration of decisions cannot simply be reduced to a set of parameters, a model of tool selection in PD still has its relevance and significance as it provides a roadmap that encourages strategic thinking in decisionmaking and serves as a checklist for policy learning. Recognizing the human elements and contextual variances in PD decisions, the model in this article is developed to be adaptive to users, purposes, and contexts.

\section{Discussion of tool selection in public policy}

This model has benefited from the discussion of tool/ instrument selection in public policy. An extensive literature has contributed to this topic from various aspects including contexts, process, attributes, and consequences of tool selection. Mayntz (1983) emphasizes the interaction and fitness between contexts and tools. Linder and Peters (1989) discusses how different levels of contexts, from the individual level of cognitive factors to institutional and systematic contexts influence the tool choice. Schneider and Ingram (1993) similarly focuses on the perceptions of individuals and further discusses people's role in meaning making process from the perspective of social constructionism. It is also implied that actors in decision-making process is incapable of acquiring all the information but need to rely on their judgments and heuristics (Jones 2001).

Specifically, many scholars have provided insights into the attributes, dynamic, and consequences of utilizing the tools (Dahl and Lindblom 1953; Ripley 1966; Salamon 2002). These different typologies of tool attributes/dimensions tend to categorize the tool options from the following aspects:

- Government presence and input (described as directness or resource intensiveness).

- Reliance on existing policy/system/market (described as automaticity or targeting).

- Support and opposition (described as political risk or visibility).

- Restriction on the behaviors of participants (described as coerciveness, intrusiveness, or constrains).

The key to tool selection is the tradeoffs and balancing acts among these attributes which produce consequences in effectiveness, efficiency, and feasibility (Salamon 2002). For example, a coercive tool is more likely to achieve accurate results but less likely to earn political support (Ripley 1966). An automatic tool (e.g., those rely on tax system or market) is easier to manage and operate, but the achievement of purposes is less certain (Diver 1983). However, corollaries of tool attributes like that are debatable in different societies, governance modes, and national cultures, and these attributes are insufficient to characterize the tools of PD. Therefore, instead of directly transplanting these attributes, this article builds on them and creates an open category of tool attributes for PD that is mindful about contextual variances and people's role in the meaning making process.

\section{From tools to flagship: toward an adaptive model of tool selection}

Drawing on knowledge from public policy and PD, this article presents an adaptive model (Fig. 1) to make sense of tool selection in flagship. Such a model is needed because a flagship needs meticulous coordination and strategic planning to optimize resource allocation among programs, balance the interests of stakeholders, and align program objectives with national goals. This model provides a method to collect and organize the data needed for these decisions in flagships. The 


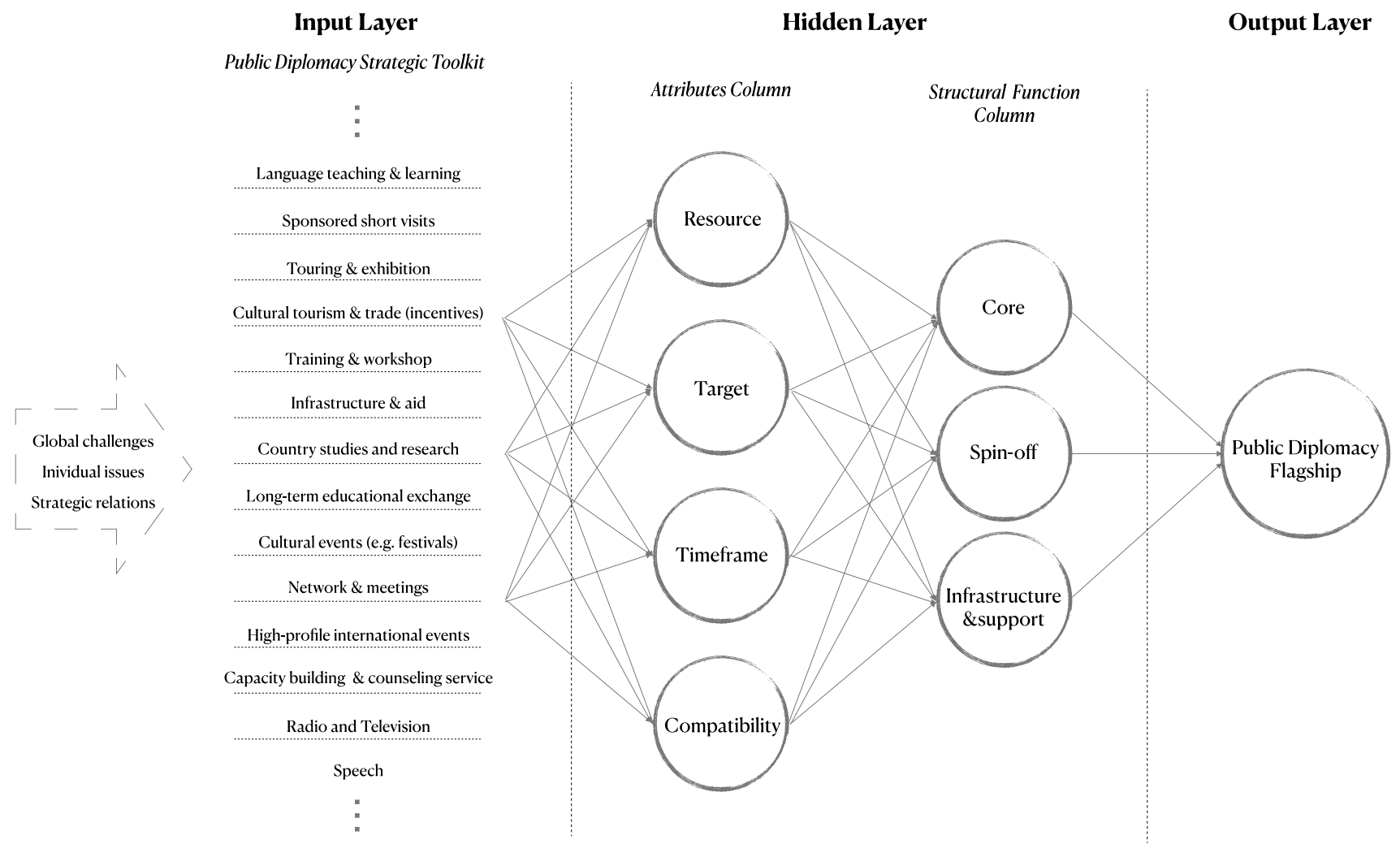

Fig. 1 An adaptive model of tool selection for public diplomacy flagships

decision of tools is driven by environmental factors including global challenges (e.g., COVID and populism) and country-specific issues (e.g., development and stability) as well as strategic relationships, and these influences persist throughout the selection process. In Fig. 1, the toolkit of PD (left) is considered as the input of tool selection and the flagship is the final output (right). The selection of tools is achieved by filtering the tool choices with the descriptive parameters in the hidden layer (middle). The key to this adaptive model is that the users can adjust the number, content, and weight of the parameters to customize the selection process.

This model assumes that the users of the model are interested in searching appropriate tools for a situation or defined problem and are willing to interact with the model to make decisions. The structure of this model is informed by Artificial Neural Networks (ANNs), first discussed by McCulloch and Pitts (1943) and now applied to fields of machine learning. The ANNs are organized into multiple connected layers to present the underlying relationships in decision making. It was developed to teach machines to imitate the human brain to build a network that adapts to changing input and generates the best possible results. This research finds the logic enlightening to guide and reflect human decisions in complex environments. As shown in Fig. 1, each neuron/circle in the hidden layer indicates a decision point or parameter that fillers or specifies a tool choice in PD. Honoring human judgement in decision making, these parameters can be manually selected and adjusted. In this article, I present a set of parameters based on my understanding of PD to capture the attributes of tools and their interrelationship in public diplomacy flagship. A brief description of each layer is presented below.

\section{Input layer: a tentative toolkit of public diplomacy}

Many studies construct taxonomies to further our understanding of public diplomacy. These taxonomies are established based on various grounds. For example, continuum and spectrum are popular formats to present the relationship between various PD activities. Evans and Steven (2010) divide the activities into four types: engagement, shaping, disruptive, and destructive on a continuum from consensual to controlling. Zaharna (2009) creates a spectrum based on two complementary views of communication: information dissemination and relationship building. In a different approach, Cull (2008, p. 35) breaks down PD activities into five components based on timeframe, information flow, and infrastructure. Similarly but with different foci, Gilboa 
United Kingdom

The British Council

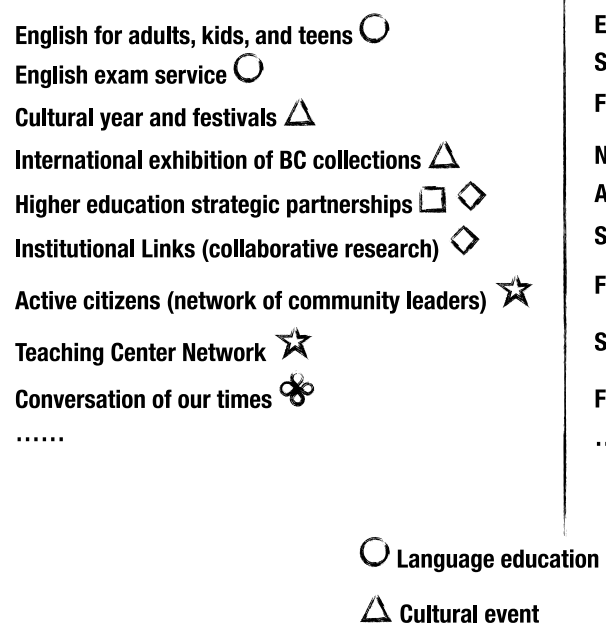

\section{United States The Fulbright Program}

English Teaching Assistant Program (a part of Student Program) $O$

Fulbright Teacher Exchanges $\square$

National Geographic Storytelling Fellowship \& $\diamond$

Arctic Initiative (policy issue research- a part of Scholar Program) $\diamond$

Fulbright American Studies Award $\diamond$

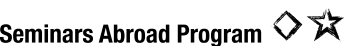

Fulbright Association

.....

Fig. 2 A sampling of programs in the British Council, the Fulbright Program, and Confucius Institute

(2008, p. 72) characterizes PD with a framework based on immediate, intermediate, and long-time dimensions that entail different purposes and means. In addition, focusing on the strategic process of PD, Banks (2020, p. 64) proposed a typology of five activities: listening, informing, advocating, connecting, and empowering. Sevin (2017, p. 891) categorizes the strategic process into six "pathways of connection" via a three-layer framework. Each of these taxonomies has its advantages and utilities in research and practice, but they are insufficient to construct an adaptive tool selection model for flagships because they focus on individual PD activities instead of the process to pick and combine the tools, and the taxonomies have limited transferability and flexibility in contexts of different cultural, political, and institutional settings.

The toolbox proposed in this article differs from the taxonomies in the following ways. A primary function of the taxonomies is to summarize and distinguish various activities or measures of PD, but the toolbox in this model is designed to present a wide range of possibilities for further adjustment or appropriation as argued in Pamment (2014, p. 54). This difference in functionality brings two distinctive features. First, the toolbox has greater inclusivity and openness compared to traditional taxonomies. Diversity is prioritized over mutual exclusivity in this toolbox. For example, educational exchange and language education can overlap and share functional similarities in practice, but they are presented as independent categories in the toolbox to provide more options as PD input and stimulate strategic thinking in decision-making. For this reason, the toolbox is constantly evolving with the contexts and experiments of users. For example, the innovation and integration of digital platforms in PD have expanded the toolbox. Since the COVID-19, virtual information exchange is replacing in-person contact at a faster rate. Such an open toolbox can better capture and reflect changes compared to traditional taxonomies. Second, this toolbox does not reflect specific values or evaluative standards but is designed to accommodate purposes and contexts of different kinds. Instead of searching for best practice, this toolbox and model seek to understand and describe the relationship between people, time, place, and PD practice.

A strategic PD toolkit of 14 categories is presented in the input layer in Fig. 1. This toolkit is further explained with examples from three flagships based on their annual reports. ${ }^{3}$ The following figure presents a sampling of the programs to illustrate the variety and relationship of the tools in the flagships.

The keys in Fig. 2 show six popular tool categories identified in the strategic toolkit. It is observed that the same tool can be adapted according to objectives and contexts. Language Education as a common PD tool provides such an example. In the Fulbright Program, English education is a part of the English Teaching Assistant Program administered by the U.S. State Department and binational commissions/embassies which embody shared interests and responsibilities; The British Council commercializes English education programs through local partnerships,

\footnotetext{
3 The annual reports include British Council (British Council 2019a), The Fulbright Program (FFSB 2017), and Confucius Institute (Hanban 2018).
} 
Table 1 Attributes of public diplomacy tools and related questions

\begin{tabular}{lll}
\hline & Related questions & Tradeoffs in capacities \\
\hline Resource & What resources are needed? & Legitimacy \\
& Who owns these resources? & Visibility \\
& What is the cost of mobilizing these resources? & Efficiency \\
Target & Who are the participants/audience? & Sustainability \\
& What is the scope of program outreach? & Accessibility \\
Time & How does the tool interact with the target? & \\
& What is the timeframe of this tool? & \\
Compatibility & How does the timeframe serve to different interests? & \\
& Is the tool appropriate for governance structure? & \\
& Is the tool consistent with national culture and values? &
\end{tabular}

and the income of which in return supports the flagship's charitable activities; Confucius Institute provides Chinese language education through the partnership between Chinese and foreign universities, which is funded and supervised by Hanban. These adaptations of tools are a major step to blend power structure and political culture into the PD flagship design. In addition to tool appropriation and adaptation, the variety of tools allows the flagships to pursue differentiated strategies in different regions. For example, the British Council prioritizes cultural partnerships in the European countries through a series of Creative Europe programs that invested in creative sectors including film, television, new media, and games. In comparison, forging educational connections as the focus in high-growth countries such as China and India brings high economic return (British Council 2019b). In Asia, the growth of British Council's exam service contributed to $£ 727$ million income growth in 2018-2019 (British Council 2019a, p. 81).

Several convergences of tool selection are also identified from Fig. 2. First, increasingly diversified and complex tools are packed together to address multi-purpose shaped by global and domestic environments. All six tool types are widely used in flagships. Many other tools listed in the strategic toolkit, such as capacity-building activities and high-profile international events are also the common practice of flagships to support their educational and cultural programs. More examples will be given in the following sections. Second, multi-actor participation expands through formal and informal relationships between state and non-state actors. The operationalization of the tools relies on individuals and organizations as both practitioners and influencers. Third, network events, the Internet, and new media as tools are applied to strengthen and rejuvenate traditional activities. These convergences indicate trends in PD as well as show a pattern of flagship tool selection that favors adaptability and transferability to broaden the international influence.

\section{Hidden layer: a strategic menu}

The hidden layer in the model offers a strategic menu for tool selection and provides insights into the two questions posed at the beginning of this article: Why are these tools selected, and how are they fitted into a flagship? To answer the questions, this layer presents two sets of parameters. The first column of parameters creates a profile for a given tool as input. This profile can contain any number of attributes. This work selects four based on public policy and PD literature and practice. The second column further processes the decision from the first column by considering whether the selected tools can fit together as a flagship and how. Depending on case scenarios, additional column(s) can be added to this hidden layer to provide more specific contexts of decision-making.

\section{Column 1: attributes of public diplomacy tools}

This article proposes four attributes that describe a tool in the toolkit: resource, target, time, and compatibility. The attributes are intended to capture the variances of tool options and invite policymakers to consider the implications of their choices. Each of these attributes can be assigned with different weights based on specific problem and situation. Table 1 lays out the questions and capacities associated with the attributes. The preference of each tool attribute is influenced by one's values, beliefs, and standards regarding the following capacities of public diplomacy flagship: legitimacy, visibility, accessibility, efficiency, and sustainability.

These capacities are prioritized differently by stakeholders with different roles such as sponsor, manager, partner, client, and participant (Fig. 3). A stakeholder can take one or multiple roles. While stakeholder is not the focus of this article, a brief discussion can explain how 


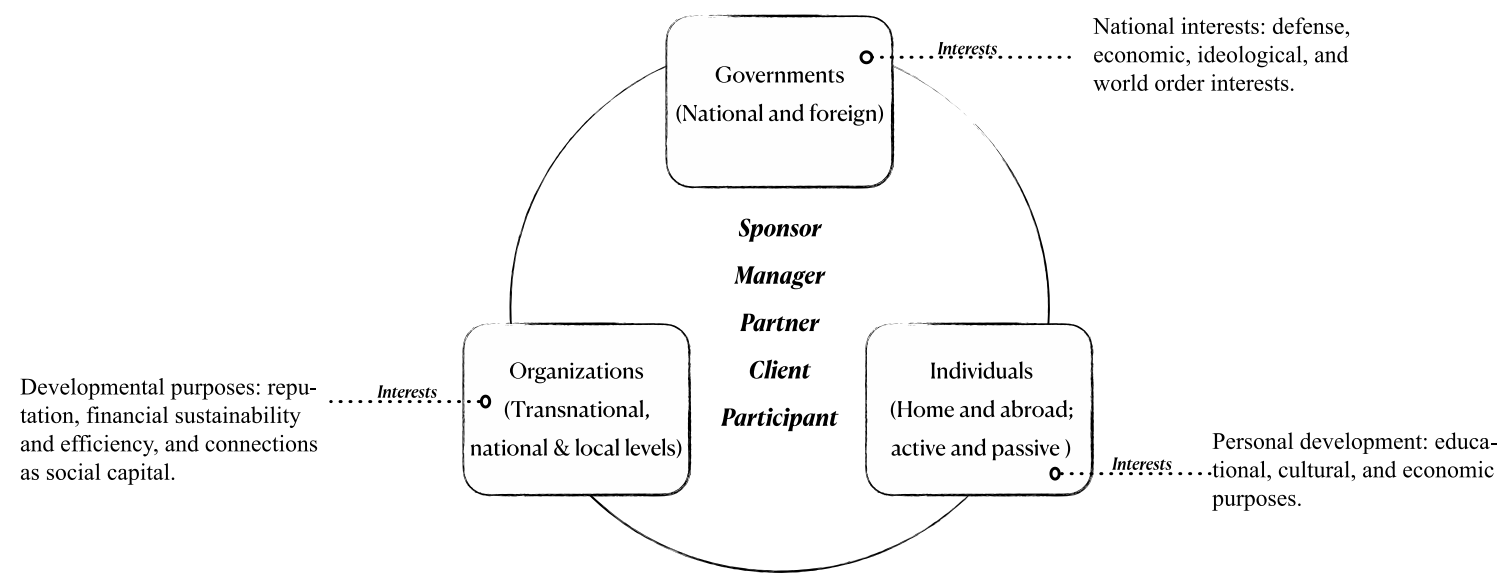

Fig. 3 Stakeholders of public diplomacy flagship

actors can approach tool selection questions differently and bow this model can be utilized by stakeholders. The government as the sponsor is predominantly concerned with the issues of legitimacy and international visibility. Thirdparty agents as the manager and cooperating organizations as the partner emphasize efficiency and sustainability in their program operation. Participants/clients value the experience and growth brought by program accessibility. The stakeholder with multiple roles could be challenged by potential conflicts and trade-offs among these capacities. For instance, the UK government is the sponsor of the British Council to provide grant-in-aid as well as the partner of the flagship to delivery programs through contracts in many fields. This dual relationship is managed and justified by a Dual Role Guidance (DRG) ${ }^{4}$ to avoid conflict of interests. Thus, to utilize the model, stakeholders with various roles can be encouraged to apply the model based on their priorities individually and then bring their results together for communication and negotiation. Specifically, the following tool attributes of tool selection are discussed in this article:

\section{Resource}

Different tools draw on resources of different forms, sources, and costs. The resources of PD are not simply monetary, but also intangible resources such as authority and knowledge. These resources are owned by various actors and are increasingly in the hands of non-state actors. The cost of acquiring resources differs in societies. For example, when sending

\footnotetext{
4 The full name of the document is British Council dual role guidance $(D R G)$ on how to avoid conflicts of interest relating to the dual roles of policy support and programme delivery for HMG-funded activity (British Council 2018).
}

an international tour/exhibition, the U.S. government often collaborates with non-profit organizations who own the cultural resources. In China, the resources are likely to be found within the public system because the state owns a fair amount of cultural production and distribution, which simplifies the planning and negotiating process and minimizes the costs in operationalizing the flagship, but the control over the resource can discourage broad societal participation and produce legitimacy concern. In addition to the considerations of control over resources, the level of investment is another trade-off faced by government. For instance, highprofile international events such as Expo and Olympics can be an ideal tool for some countries considering the international visibility and reputation it can produce in a short time with one-time investment. However, for some others, the costs and efforts of mobilizing the resources for such an event can be significant and not cost-effective. This "relative disadvantage" of mega-events can outweigh all other considerations and make them unfeasible as a tool option.

\section{Target}

The target of PD can be viewed in two groups: domestic constituency and foreign publics. Whether the tool choice is compelling to constituency influences the legitimacy and sustainability of a flagship, which will be further discussed in the compatibility section. More commonly, foreign publics is referred as the target of PD. Each tool reaches and serves populations with different characteristics in age, professions, geography, etc. For example, sponsored short visits such as summer camps for educational purposes typically attract young students, whereas research and country studies projects sponsor senior scholars. The size of the population targeted by a tool also varies. For example, television programs are offered to broad audience without differentiation and segmentation, while exhibitions and performances 
target a small group of audience with certain knowledge and background to offer more immersive experience. In order to match a tool to appropriate audience, research on PD targets (Fitzpatrick 2012; Tam and Kim 2019) is useful in understanding the connections between public behaviors, environments, and PD strategies. Tradeoffs in this parameter center on the breadth (accessibility) and depth (customized immersive experience) offered by a tool in the flagship. Although there is no best universal design, a multi-tool portfolio offers advantages over a single tool choice by balancing accessibility and interactivity among the tools.

\section{Time}

PD tools are operated in different timeframes. The tools primarily for advocacy purposes usually operate in short periods, such as speeches and official posts on social media platforms. In contrast, repetitive, intergenerational communication and research projects are tools with longer time horizon. A fundamental difference found between short-term and long-term oriented programs is how individual goals are aligned with government agenda. In long-term programs, government agenda is served indirectly through the attainment of personal goals, the change of individual behavior, and multiplier effects over the time. By contrast, a short-term tool, which has little time to "listen", is under pressure to demonstrate its effectiveness in serving narrow government agenda. This comparison does not exclude short-term tools as an important option in flagship for of their important role in establishing a presence and managing crises. As discussed in Gilboa (2008), Leonard et al. (2002) and (Golan 2013), short-term programs (hours and days) have the immediate purpose of being reactive; medium-term programs (weeks and months) are proactive and engage in strategic communication; long-term programs aim at relationship building. Therefore, flagships are likely to benefit from the availability of multiple tools with different timeframes in order to address different situations.

\section{Compatibility}

Ideology, political regime, and governance structure of a country could favor certain tools over others. For example, in the United States, reciprocal exchange programs are likely to sustain its public support because they are consistent with national values and widely engage individuals and organizations to share benefits compared to international broadcasting or foreign aid programs. However, in a culture that supports strong government, a PD tool that directly demonstrates the strength and international influence of the country could be more popular among the public as it satisfies the expectation of a powerful state. The Belt and Road Initiative of China is such an example. Additionally, policy legacies and established governance structure could influence tool selection decisions by narrowing the number of choice through time, creating a self-reinforcing process described as path dependency (Pierson 2000). This implies that if a tool of PD had been successful in the past, policymakers incline to rely on the same mechanism for future problems. International educational exchange has been such a versatile and proven reliable tool of PD, welcomed by both constituency and foreign publics. Compatibility and path dependency seem to be two sides of same coin. While an old tool could be a safe option considering popularity and efficiency, a path dependent mentality can be dangerous in the long run. As PD becomes increasingly ad hoc and spontaneous (Snow 2014), a single and fixed choice of tools will hinder the competitiveness of flagships.

These four attributes present an array of options and questions in front of policymakers and analysts, and there are more to be discovered. Different approaches can be taken to utilize the parameters in this column. A desirable tool profile could be created by articulating the preferences in each attribute, and then the profile can be used as a filter to examine the tool options from the toolkit. Alternatively, without committing to a specific configuration of attributes, the column could be used to compare the tools and explore their differences and implications.

\section{Column 2: structural functions of public diplomacy tools}

Column 2 in the hidden layer is designed to parse the structural function of selected tools. The structural function describes the role played by a tool in structuring the flagship. In other words, it describes how a tool relates to other tools in a flagship. Inspired by the experience of flagships, this article presents one of the many ways to structure a flagship by dividing the structural functions of PD tools into three groups: core programs, spin-off programs, and support and infrastructure programs. Figure 4 illustrates the structural functions of tools and their relationship in a flagship.

A core program is the primary focus/pillar of a public diplomacy flagship. It is usually created at an early stage and continues to grow with the flagship. The core program embodies the values of a flagship and best represents its mission. For the Fulbright Program, the initial exchange programs authorized by the Fulbright Act of 1946 has been the core of the flagship. This format of reciprocal exchange continues to today and becomes the foundation for newly created programs in the flagship. For the British Council and Confucius Institute, language education programs as the core have built up the reputation for the flagships and removed the communication barriers for other programs under the flagships. In conjunction with the first column, the core program has the following attributes. First, it requires 


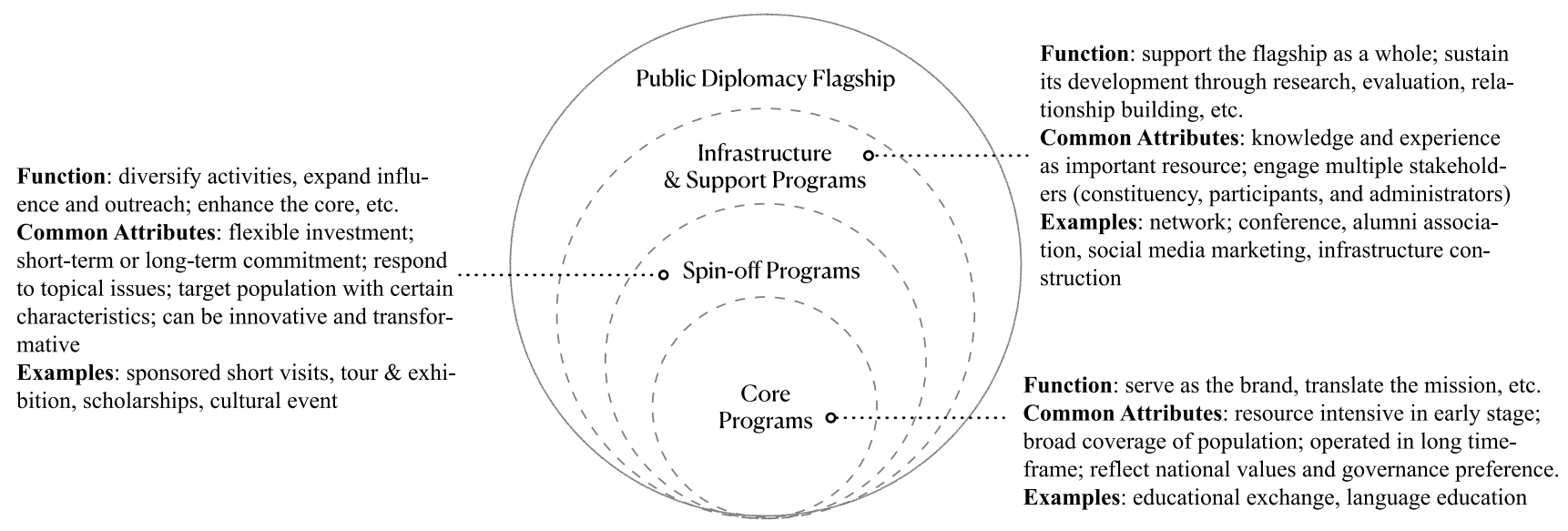

Fig. 4 Structural functions of tools in public diplomacy flagship

significant government input at the beginning to establish the structure to operationalize the program and make connection with target population. This usually means establishing physical presences in foreign countries. The Fulbright Program established Binational Commissions to run scholar exchange programs. The British Council connects with foreign countries through overseas representatives (business or nonprofits), and Chinese Confucius Institute establishes organizations and classrooms in foreign universities. These physical presences and structures, as the core programs expand, have become networks that broadly serve the entire flagship. Second, these core programs have a broad outreach covering vast geographic locations and are operated with long timeframe. These core programs become the window for foreign publics to learn about the flagship and the country. Across generations, these core programs are the key to legitimize the flagship through its continuity and accessibility while many other programs under the flagship can be ad hoc and transitional to address a particular issue at a time or in a particular region. For example, the Fulbright's New Century Scholars Program was operated between 2001 and 2010 to address specific global issues through interdisciplinary and collaborative research across borders. The research topics were assigned annually. In 2002-2003, just after 9/11, the topic was "Addressing Sectarian, Ethnic and Cultural Conflict within and across National Borders," which marks an immediate Fulbright response to terrorism (FFSB 2002). These short programs increase the adaptability and flexibility of the flagship through timely response and visible results in a short time. The core programs, on the other hand, contribute to consistency and stability through long-term relationship building.

Spin-off program is closely related to core program and provides services and experiences in broader fields. "Spinoff" does not imply it is less important than the core but suggests that these programs have greater diversity and coverage beyond the core focus. The spin-off programs are usually layered and packed with core programs to enlarge and deepen the impacts of flagship. They do not necessarily have a long-time commitment, and many of these programs target only a slice of the population. The aforementioned New Century Scholars Program is an example. Confucius Institutes have developed many spin-off programs around its core of Chinese language education. For instance, Confucius Institute Scholarship program as a spin-off program helps to remove barriers for students to participate in language education programs. Online and Air Confucius Institutes use broadcasting and the Internet as tools to provide a second channel for the Chinese language learners to access teaching and learning materials. Chinese Bridge, a competition for Chinese language and culture, is another cultural spin-off program that aims to facilitate Chinese learning and understanding about China (Hanban 2018). Cultural events and sponsored short visits are also common spin-off program options because they are flexible and adaptable to various topics, time length, and audience. The spin-off programs connect to the core but enjoy greater autonomy regarding program content and format: They diversify tool selections and form a healthy relationship with the core, enhancing the appeal of the flagship.

Lastly, infrastructure and support program is designed to provide resources and improve the capacities and reflexivity of the flagship. A typical tool with this structural function is meetings and networking events that connect decisionmakers, administrators, and participants. Meetings and networks can support a flagship in several ways. First, it helps to improve the capacities of the flagship through personal development and knowledge transfer. As Zaharna (2005) points out, network can mitigate problems created by information dominance and asymmetry. The Teaching Centre Network of the British Council provides an example. The network engages 50 countries through a consistent 
accreditation approach to share experience and promote best practice in English teaching (Foreign \& Commonwealth Office 2019). Second, these network events provide flagships with opportunities to reflect, evaluate, and improve through communication with stakeholders at different levels. The annual conferences of public diplomacy flagships often serve this purpose to bring multiple stakeholders together. For example, Confucius Institute Annual Conference invites university presidents and Confucius Institutes representatives as well as officials from headquarters and State Council to reflect on and discuss the opportunities and challenges (Hanban 2018). Third, the infrastructure and support programs advocate for continuing support for flagships. Conferences, alumni associations and networks take this role to mobilize resources. The Fulbright Association organizes alumni through local chapters and annual conferences, which builds an active constituency to educate the Congress and the public about the Fulbright program and helps to secure the appropriation for the flagship. Other options for infrastructure and support programs include physical infrastructure such as the construction of cultural centers in foreign countries as both a venue for cultural events and a foreign investment. Confucius Institute in belt and road countries is such an example. In addition, research departments or divisions become a common part of flagships to evaluate and advise on the planning and implementation of the flagship. For example, Chinese Confucius Institute has created a research program to develop teaching materials and methods to support other programs. In brief, the programs with this structural function provide resources, tangible or intangible, to the flagship as a whole and sustain its development through research, advocacy, network, etc.

The above discussion of core programs, spin-off programs, and infrastructure and support programs introduced a simplified yet expandable structural relationship in public diplomacy flagships. Depending on scale, purposes, and even developmental stages, a flagship could have more than one core program, and each core can have its own spin-off and support programs or share the programs with another core. For example, throughout the years, the British Council has developed into a multi-core flagship where the programs and services are grouped into three strategic business units: Arts, Education and Society, English and Exams (British Council 2016). Each of the units includes a set of mutually supported programs with different attributes. Thus, the relationship between the tools evolves with the functions of the tools, which is appropriated by the tool attributes under the influence of changing environments and interests. Clarification and strategic planning of structural functions can facilitate the communication and collaboration between programs and enhance the sustainability and reflectivity of flagship.

Column 1 of tool attributes and column 2 of structural functions are closely linked in tool decisions, yet they process information from different perspectives. While tool attributes deconstruct each individual tool to provide reference for decision-making, structural functions bring isolated tools together as one flagship. In other words, the first column discusses whether a tool is a feasible one, and the second column further considers if it is an appropriate tool in a given flagship system. Most importantly, when tradeoffs arise, discretion and judgement driven by accountability, experience, and expertise can play a greater role in tool selection than the criteria themselves. These two columns from the hidden layer interpret how the toolkit as the input can be translated into a public diplomacy flagship as the output. The model offers one interpretation among many possibilities to highlight that the selection/decision in a flagship requires a more systematic approach that allows policymakers and researchers to navigate through the complexities and uncertainties and address multiple purposes with limited resources.

\section{Summary and application scenarios}

This model describes the decision-making process for selecting and adapting the tools of PD and structuring them into a flagship that is capable of addressing the multiple interests of various stakeholders in a fluid environment. Inspired by the neural network model, this model is attentive to the underlying relationships in decision-making process and divides the process into three layers: input, hidden, and output layers. The input layer is a toolkit of PD, and the output layer is the flagship. The hidden layer, as the focus of this work, seeks to propose a strategic menu for PD tool selection. Two sets of parameters are presented in two columns to fill in the gap between tool options and a complete flagship. These two columns are interconnected and aim to address two questions: why or why not a tool is selected by policymakers given specific tool attributes? How do these tools fit together as a flagship? This article discussed four attributes of PD: resource, target, timeframe, and compatibility. Based on this, a structural relationship is discussed to organize the tools into three groups with different structural functions (core, spin-off, and infrastructure and support program) in a flagship.

The model is characterized by its adaptability to both users and methods. Policymakers, analysts, scholars, and students, as users, can customize the model for their analytical, evaluative, and comparative purposes. The adaptability is achieved by recognizing human factors and allowing their interaction with the model, which complements the limitation of generalization. The model is structured, but also reserves room for discretion and judgment based on preferences, purposes, and experiences. Users can expand or reduce the parameters and assign different priorities/weights 
to each parameter. The adaptability of this model is also reflected in its openness to qualitative and quantitative methods. For example, in discourse analysis and content analysis, this model can assist discussion at both conceptual and relational levels by providing a preliminary coding scheme or categories. Using this as a starting point, the relationship between PD tools and their roles in a flagship can be investigated. In addition to retrospective analysis, this model also has the potentials in prospective analysis that can improve decision making. Since this multi-layer selection model is inspired by the ANNs which is already used as a support system for managerial decisions (e.g., in financial and environmental issues), there is a possibility to adjust the model and combine it with algorithms to support decisions in PD. However, regardless of user or method, the human component of this model is as important as the role of people in PD activities. This model is not intended to limit the options, but rather to present more possibilities in public diplomacy.

\section{Declaration}

Conflict of interest On behalf of all authors, the corresponding author states that there is no conflict of interest.

\section{References}

Anholt, Simon. 2006. Public Diplomacy and Place Branding: Where's the LINK? Place Branding 2 (4): 271-275.

Robert, Banks. 2020. Public Diplomacy Evaluation. In Routledge Handbook of Public Diplomacy, ed. Nancy Snow and Nicholas J. Cull, 64-75. London: Routledge.

Bianchini, Franco, Jon Dawson, and Richard Evans. 1992. Flagship Projects in Urban Regeneration. In Rebuilding the City: PropertyLed Urban Regeneration, ed. Pasty Healey, Simin Davoudi and Solmaz Tavsanoglu, 245-255.

British Council. 2016. Appraisal Report, 1934-2016.

British Council. 2018. British Council Dual Role Guidance (DRG) on How to Avoid Conflicts of Interest Relating to the Dual Roles of Policy Support and Programme Delivery for HMG-Funded Activity.

British Council. 2019a. Annual Report and Accounts 2018-19. London: British Council.

British Council. 2019b. Corporate Plan 2019-20. London.

Cull, Nicholas J. 2008. Public Diplomacy: Taxonomies and Histories. The Annals of the American Academy of Political and Social Science 616 (1): 31-54.

Dahl, Robert Alan, and Charles Edward Lindblom. 1953. Politics, Economics and Welfare: Planning and Politico-Economic Systems, Resolved into Basic Processes. New York: Harper \& Brothers.

De Frantz, Monika. 2005. From Cultural Regeneration to Discursive Governance: Constructing the Flagship of the 'Museumsquartier Vienna' as a Plural Symbol of Change. International Journal of Urban and Regional Research 29 (1): 50-66.

Diver, Colin S. 1983. The optimal precision of administrative rules. Yale Law Journal 93: 65.

Evans, Alex, and David Steven. 2010. Towards a Theory of Influence for Twenty-First Century Foreign Policy: The New Public
Diplomacy in a Globalized World. Place Branding and Public Diplomacy 6 (1): 18-26.

FFSB. 2002. Thirty-Ninth Annual Report. Washington, DC.

FFSB. 2017. J. William Fulbright Foreign Scholarship Board Annual Report.

Fitzpatrick, Kathy R. 2012. Defining Strategic Publics in a Networked World: Public Diplomacy's Challenge at Home and Abroad. The Hague Journal of Diplomacy 7 (4): 421-440.

Foreign \& Commonwealth Office. 2019. British Council Tailored Review.

Gilboa, Eytan. 2008. Searching for a Theory of Public Diplomacy. The Annals of the American Academy of Political and Social Science 616 (1): 55-77.

Golan, Guy J. 2013. An Integrated Approach to Public Diplomacy. Los Angeles, CA: Sage Publications Sage CA.

Grodach, Carl. 2010. Beyond Bilbao: Rethinking Flagship Cultural Development and Planning in Three California Cities. Journal of Planning Education and Research 29 (3): 353-366.

Hanban. 2016. Confucius Institute Annual Development Report. Beijing.

Hanban. 2018. Confucius Institute Annual Development Report. Beijing.

Hocking, Brian, Jan Melissen, Shaun Riordan, and Paul Sharp. 2013. Futures for Diplomacy: Integrative Diplomacy in the 21st Century. Clingendael Netherlands Institute of International Relations.

Jones, Bryan D. 2001. Politics and the Architecture of Choice: Bounded Rationality and Governance. Chicago: University of Chicago Press.

Leonard, Mark, Catherine Stead, and Conrad Smewing. 2002. Public diplomacy: Foreign Policy Centre.

Linder, Stephen H., and B. Guy Peters. 1989. Instruments of Government: Perceptions and Contexts. Journal of Public Policy 9 (1): $35-58$.

Mayntz, Renate. 1983. The Conditions of Effective Public Policy: A New Challenge for Policy Analysis. Policy \& Politics 11 (2): 123-143.

McCulloch, Warren S., and Walter Pitts. 1943. A Logical Calculus of the Ideas Immanent in Nervous Activity. The Bulletin of Mathematical Biophysics 5 (4): 115-133.

Melissen, Jan, and Jian Wang. 2019. Debating Public Diplomacy: Now and Next. Boston: BRILL.

Pacher, Andreas. 2018. The Ritual Creation of Political Symbols: International Exchanges in Public Diplomacy. The British Journal of Politics and International Relations 20 (4): 880-897. https://doi. org/10.1177/1369148118786043.

Pamment, James. 2014. Articulating Influence: Toward a Research Agenda for Interpreting the Evaluation of Soft Power, Public Diplomacy and Nation Brands. Public Relations Review 40 (1): 50-59.

Pierson, Paul. 2000. Increasing Returns, Path Dependence, and the Study of Politics. American Political Science Review 94: 251-267.

Ripley, Randall B. 1966. Public Policies and Their Politics: Techniques of Government Control. NewYork: W. W. Norton.

Salamon, Lester M. 2002. The Tools of Government: A Guide to the New Governance. Oxford: Oxford University Press.

Schneider, Anne, and Helen Ingram. 1993. Social Construction of Target Populations: Implications for Politics and Policy. American Political Science Review 87 (2): 334-347.

Sevin, Efe. 2017. A Multilayered Approach to Public Diplomacy Evaluation: Pathways of Connection. Politics \& Policy 45 (5): 879-901.

Sevin, Efe, and Diana Ingenhoff. 2018. Public Diplomacy on Social Media: Analyzing Networks and Content. International Journal of Communication 12: 23.

Smyth, Hedley. 2005. Marketing the City: The Role of Flagship Developments in Urban Regeneration. London: Taylor \& Francis. 
Snow, Nancy. 2014. Public Diplomacy: New Dimensions and Implications. In Global Communication: Theories, Stakeholders, and Trends, ed. Thomas L. McPhail, 64-79. Blackwell: Wiley.

Tam, Lisa, and Jeong-Nam. Kim. 2019. Who are Publics in Public Diplomacy? Proposing a Taxonomy of Foreign Publics as an Intersection Between Symbolic Environment and Behavioral Experiences. Place Branding and Public Diplomacy 15 (1): $28-37$.

Zaharna, Rhonda S. 2005. The Network Paradigm of Strategic Public Diplomacy. Foreign Policy in Focus 10 (1): 1-4.

Zaharna, R.S. 2009. Mapping Out a Spectrum of Public Diplomacy Initiatives. In Routledge Handbook of Public Diplomacy, ed. Nancy Snow and Philip M. Taylor.
Publisher's Note Springer Nature remains neutral with regard to jurisdictional claims in published maps and institutional affiliations.

Biyun Zhu received her PhD from The Ohio State University in 2021. Previously, she earned a master's degree in Public Policy from King's College London and a bachelor's degree in Public Administration from the University of International Business and Economics. Her research interests include public diplomacy, cultural diplomacy, and cultural policy. Her recent work has focused on multi-level policy design of cultural diplomacy in great power countries. 